\title{
Editorial
}

\section{Neutrophil Gelatinase-Associated Lipocalin as a Promising Novel Biomarker for Early Detection of Kidney Injury}

Acute kidney injury (AKI) is a common complication of various critical illnesses and shows independent associations with mortality, morbidity, and prolonged hospitalization [1-5]. The current standard of the diagnostic criteria for AKI is based on consensus from consortiums such as the AKI Network or Kidney Disease: Improving Global Outcomes (KDIGO) [6]. Their AKI definitions involve increased serum creatinine (SCr) and/or decreased urine output $[5,6]$. This SCr-based standardization provides several merits from both research and clinical aspects by permitting comparative analysis among patients or studies [1]. However, there are some recognized limitations of these SCr-based AKI definitions as outlined below [2, 3].

The first limitation is that an increase in SCr does not perfectly match with the degree of structural kidney injury. For example, hemodynamic reduction in renal perfusion can cause increasing SCr levels without definite structural renal impairment [1]. In addition, renin angiotensin aldosterone system inhibitors, which are among the first-line antihypertensive medications, can also result in a SCr increase without accompanying structural kidney injury [7]. The second limitation is that kidney injury can occur without an increase in $\mathrm{SCr}$ levels. A renal reserve phenomenon is a representative example of this effect [1]. Functionally reserved parts of nephrons substitute for the injured tubules and reduced glomerular filtration rate (GFR), especially in the early phase of the continuous spectrum of renal impairment [1]. This renal reserve phenomenon results in a lag period of about two three days after the start of kidney injury until an SCr increase is detected [1]. This delay may lead to loss of the optimal chance for the early detection of structural kidney injury and the immediate start of appropriate treatment [1]. Besides, a low muscle mass, which leads to decreased creatinine production, can also hinder the detection of structural kidney injury, particularly in newborns or premature babies who have only a very small muscle mass [8].

To overcome these well-known limitations of SCr, novel biomarkers for AKI have been diligently explored to date [2]. Within the last two decades, several new renal markers that are measurable in the blood or urine have been proposed, including neutrophil gelatinase-associated lipocalin (NGAL) [5, 9-11]. NGAL, also known as siderocalin or lipocalin 2, is a $25-\mathrm{kDa}$ protein in the lipocalin family [5]. NGAL exhibits a bacteriostatic function by binding to and sequestering the iron-siderophore complex, which hinders the bacterial utilization of iron [12]. NGAL is slowly and continuously expressed in various human tissues, including the lung, stomach, prostate, uterus, and kidney [5]. However, the kidney shows particularly upregulated NGAL expression after toxic, septic, or ischemic injuries, especially from the collecting duct and the thick ascending limb of the loop of Henle [2, 5]. Therefore, NGAL is regarded as an early biomarker of renal tubular damage. After the initial insult, its increase can be detected within 3 hours, reaching a peak at about 6-12 hours, and lasts for up to five days [5].

Accordingly, NGAL is one of the most thoroughly and extensively studied renal markers for heterogeneous phenotypes of 
$\mathrm{AKI}$, including those associated with nephrotoxic and ischemic injuries $[1,4,11,13,14]$. Recently, the field of research related to NGAL has broadened beyond AKI, including investigations of its association with chronic renal diseases such as diabetic nephropathy or various infectious conditions such as urinary tract infection [2, 10, 14]. In this issue of Annals of Laboratory Medicine, we introduce an original study that evaluated NGAL in febrile urinary tract infection [9]. These trials for marginal expansion of the clinical adaptation of NGAL may solidify the position of NGAL as a promising next-generation biomarker for the early detection of renal impairment beyond AKI [11].

\section{Authors' Disclosures of Potential Conflicts of Interest}

No potential conflicts of interest relevant to this article were reported.

\section{References}

1. Moledina DG and Parikh CR. Phenotyping of acute kidney injury: beyond serum creatinine. Semin Nephrol 2018;38:3-11.

2. Kim H, Hur M, Lee S, Marino R, Magrini L, Cardelli P, et al. Proenkephalin, neutrophil gelatinase-associated lipocalin, and estimated glomerular filtration rates in patients with sepsis. Ann Lab Med 2017;37:388-97.

3. Klein SJ, Brandtner AK, Lehner GF, Ulmer H, Bagshaw SM, Wiedermann CJ, et al. Biomarkers for prediction of renal replacement therapy in acute kidney injury: a systematic review and meta-analysis. Intensive Care Med 2018;44:323-36.

4. Hjortrup PB, Haase N, Wetterslev M, Perner A. Clinical review: predictive value of neutrophil gelatinase-associated lipocalin for acute kidney injury in intensive care patients. Crit Care 2013;17:211.

5. Kashani K, Cheungpasitporn W, Ronco C. Biomarkers of acute kidney injury: the pathway from discovery to clinical adoption. Clin Chem Lab Med 2017:55:1074-89.

6. Kidney Disease: Improving Global Outcomes (KDIGO) CKD-MBD Working Group. KDIGO clinical practice guideline for the diagnosis, evalua- tion, prevention, and treatment of chronic kidney disease-mineral and bone disorder (CKD-MBD). Kidney Int Suppl 2009;S1-130.

7. Coca SG, Garg AX, Swaminathan M, Garwood S, Hong K, ThiessenPhilbrook $\mathrm{H}$, et al. Preoperative angiotensin-converting enzyme inhibitors and angiotensin receptor blocker use and acute kidney injury in patients undergoing cardiac surgery. Nephrol Dial Transplant 2013;28: 2787-99.

8. Cho SY, Hahn WH, Lee HJ, Suh JT, Lee A, Cho BS, et al. The clinical significance of serum cystatin $C$ in critically ill newborns with normal serum creatinine. J Clin Lab Anal 2012;26:267-71.

9. Yun BA, Yang EY, Kim CJ. Plasma neutrophil gelatinase-associated lipocalin as a predictor of renal parenchymal involvement in infants with febrile urinary tract infection: a preliminary study. Ann Lab Med 2018;38: 425-30.

10. Fiseha T, Tamir Z. Urinary markers of tubular injury in early diabetic nephropathy. Int J Nephrol 2016;2016:4647685.

11. Malhotra R and Siew ED. Biomarkers for the early detection and prognosis of acute kidney injury. Clin J Am Soc Nephrol 2017;12:149-73.

12. Flo TH, Smith KD, Sato S, Rodriguez DJ, Holmes MA, Strong RK, et al. Lipocalin 2 mediates an innate immune response to bacterial infection by sequestrating iron. Nature 2004;432:917-21.

13. Tong J, Li H, Zhang H, Luo Z, Huang Y, Huang J, et al. Neutrophil gelatinase-associated lipocalin in the prediction of contrast-induced nephropathy: a systemic review and meta-analysis. J Cardiovasc Pharmacol 2015;66:239-45

14. Ronco C, Legrand M, Goldstein SL, Hur M, Tran N, Howell EC, et al. Neutrophil gelatinase-associated lipocalin: ready for routine clinical use? An international perspective. Blood Purif 2014;37:271-85.

\section{Corresponding author: Sun Young Cho}

(iD https://orcid.org/0000-0002-3208-5446

Department of Laboratory Medicine, School of Medicine, Kyung Hee

University Hospital, 23 Kyungheedae-ro, Dongdaemun-gu, Seoul 02447 Korea

Tel: +82-2-958-8671, Fax: +82-2-958-8609

E-mail: untoyou@hanmail.net

\section{Co-corresponding author: Mina Hur}

(iD https://orcid.org/0000-0002-4429-9978

Department of Laboratory Medicine, Konkuk University School of Medicine, 120-1 Neungdong-ro, Gwangjin-gu, Seoul 05030, Korea

Tel: +82-2-2030-5581, Fax: +82-2-2636-6764

E-mail: dearmina@hanmail.net 